\title{
Kynurenine metabolic balance is disrupted in the hippocampus following peripheral lipopolysaccharide challenge
}

\author{
Jennifer M. Parrott' ${ }^{1}$, Laney Redus ${ }^{2}$ and Jason C. O'Connor ${ }^{3,4^{*}}$
}

\begin{abstract}
Background: Inflammation increases the risk of developing depression-related symptoms, and tryptophan metabolism is an important mediator of these behavior changes. Peripheral immune activation results in central up-regulation of pro-inflammatory cytokine expression, microglia activation, and the production of neurotoxic kynurenine metabolites. The neuroinflammatory and kynurenine metabolic response to peripheral immune activation has been largely characterized at the whole brain level. It is unknown if this metabolic response exhibits regional specificity even though the unique indoleamine 2,3-dioxygenase (IDO)-dependent depressive-like behaviors are known to be controlled by discrete brain regions. Therefore, regional characterization of neuroinflammation and kynurenine metabolism might allow for better understanding of the potential mechanisms that mediate inflammation-associated behavior changes.

Methods: Following peripheral immune challenge with lipopolysaccharide (LPS), brain tissue from behaviorally relevant regions was analyzed for changes in mRNA of neuroinflammatory targets and kynurenine pathway enzymes. The metabolic balance of the kynurenine pathway was also determined in the peripheral circulation and these brain regions.
\end{abstract}

Results: Peripheral LPS treatment resulted in region-independent up-regulation of brain expression of pro-inflammatory cytokines and glial cellular markers indicative of a neuroinflammatory response. The expression of kynurenine pathway enzymes was also largely region-independent. While the kynurenine/tryptophan ratio was elevated significantly in both the plasma and in each brain regions evaluated, the balance of kynurenine metabolism was skewed toward production of neurotoxic metabolites in the hippocampus.

Conclusions: The upstream neuroinflammatory processes, such as pro-inflammatory cytokine production, glial cell activation, and kynurenine production, may be similar throughout the brain. However, it appears that the balance of downstream kynurenine metabolism is a tightly regulated brain region-dependent process.

Keywords: Neuroinflammation, Kynurenine, Pro-inflammatory cytokines, Brain regions, Microglia, Indoleamine 2,3-dioxygenase, Kynurenine 3-monooxygenase, Hippocampus

\footnotetext{
* Correspondence: oconnorj@uthscsa.edu

${ }^{3}$ Department of Pharmacology, School of Medicine, Center for Biomedical Neuroscience and Mood Disorders Translational Research Core, University of Texas Health Science Center at San Antonio, 216B Medical Building MC-7764, 7703 Floyd Curl Drive, San Antonio, TX 78229-3900, USA

${ }^{4}$ Audie L. Murphy Memorial VA Hospital, South Texas Veterans Health

System, 7400 Merton Minter, San Antonio, Texas 78229-4404, USA

Full list of author information is available at the end of the article
} 


\section{Background}

The behavioral consequences of inflammation and pro-inflammatory cytokines have been well described, both clinically and preclinically. Chronic diseases, often characterized by prolonged immune activation, are associated with an increased risk of comorbid depression diagnosis [1]. Approximately 50-80\% of patients receiving interferon- $\alpha$ (IFN- $\alpha$ ) immunotherapy for hepatitis $\mathrm{C}$ or malignant melanoma develop depression-related symptoms during the course of treatment, including neurovegetative, affective, and/or cognitive impairment [2]. Administration of endotoxin or vaccines to healthy volunteers induces transient depression-associated symptoms that parallel the appearance of pro-inflammatory cytokines [3-6], whereas direct administration of proinflammatory cytokines in preclinical models recapitulates inflammation-associated depressive-like behaviors [7-10]. Pharmacological or genetic inhibition of proinflammatory cytokine action effectively attenuates many of the inflammation-induced depressive-like behaviors in rodents, establishing that pro-inflammatory cytokines are necessary to precipitate this behavioral response $[11,12]$. Clearly, pro-inflammatory cytokines are important pathogenic mediators of depression-related behavior changes resulting from immune stimulation; however, the specific mechanism by which this occurs is not clearly defined.

Peripheral immune activation and subsequent production of pro-inflammatory cytokines communicate immune status to the brain via multiple routes, where the inflammatory signals are locally propagated by microglia, the resident immune cells [13]. While the initial neuroinflammatory response may be part of an adaptive beneficial process, excessive or chronic neuroinflammation has been associated with adverse neurological consequences $[14,15]$. These consequences can include the disruption of neurotransmission, neurotrophin signaling, hypothalamic-pituitary-adrenal (HPA) axis activation, and alteration of metabolic pathways [13]. One metabolic pathway hypothesized to be important in mediating the effects of pro-inflammatory cytokines in the brain is the kynurenine pathway of tryptophan metabolism (Fig. 1), the major route of central tryptophan breakdown [16]. Pro-inflammatory cytokines up-regulate the expression and activity of indoleamine 2,3-dioxygenase (IDO, Fig. 1), the rate-limiting enzyme of the kynurenine pathway, increasing flux through the pathway [17-19]. During basal conditions, kynurenine is further metabolized to kynurenic acid (KA, Fig. 1), an $N$-methyl-D-aspartate receptor (NMDAR) antagonist and an $\alpha_{7-n i c o t i n i c}$ acetylcholine receptor $\left(\alpha_{7} \mathrm{nAChR}\right)$ negative allosteric modulator $[20,21]$. However, during neuroinflammatory conditions, the "neurotoxic" branch of kynurenine metabolism is up-regulated, which drives the synthesis of several neuroactive metabolites, including quinolinic acid (QA, Fig. 1), an
NMDAR agonist and neurotoxic metabolite [20, 22, 23]. This shift in kynurenine pathway balance, increasing the production of neurotoxic QA relative to KA, that occurs during neuroinflammation, is hypothesized to underlie inflammation-associated depression-related behaviors [24]. Inhibition or genetic removal of IDO demonstrated the necessity of kynurenine metabolism to the development of inflammation-induced depressive-like behaviors in rodents $[19,25,26]$. Further, antagonism of the NMDAR with ketamine attenuated inflammation-induced anhedonia and behavioral despair, implicating activation of NMDAR by QA, as a mechanism that induces inflammation-associated depressive-like behaviors [23]. Interferon- $\alpha$ immunotherapy resulted in elevated serum kynurenine/tryptophan ratio, indicative of increased IDO activity, as well as increased cerebrospinal fluid (CSF) levels of kynurenine, KA, and QA $[27,28]$. Only CSF QA was significantly positively associated with Montgomery-Asberg Depression Rating Scale (MADRS) scores in these patients [28]. However, to date, inflammation-induced changes in kynurenine metabolism have largely been measured in whole brain samples even though distinct, regionally specific neurocircuitry controls the various symptom dimensions of depression.

Depression symptomatology is heterogeneous throughout the patient population, complicating research efforts targeted at understanding the neurobiological substrates underlying the disease. Because of this, the National Institute of Mental Health (NIMH) established the Research Domain Criteria (RDoC, http://www.nimh. nih.gov/research-priorities/rdoc/index.shtml) as a construct for mental health research [29, 30]. Rather than defining patient populations by a disease diagnosis, the $\mathrm{RDoC}$ instead prompts investigators to focus on understanding the mechanism that underlies specific symptoms or behaviors. In healthy volunteers, endotoxin administration results in anhedonia, disruption in cognitive performance, anxiety, and mood disturbances $[31,32]$. These endotoxin-associated symptoms have been associated with altered blood flow (imaged using functional magnetic resonance imaging (fMRI)) in the ventral striatum, amygdala, and dorsomedial prefrontal cortex among other regions [5, 33, 34]. Similar to human patients, lipopolysaccharide (LPS, endotoxin) challenge in mice results in similar behaviors (anhedonia, anxiety, behavioral despair, cognitive disruption) $[19,25,26]$. Determining the relevant regional neuroinflammatory response, particularly kynurenine metabolism, following peripheral immune activation with LPS will lead to a better understanding of the development of these behaviors.

While the region-specific expression of pro-inflammatory cytokines after peripheral immune challenge has been partially characterized $[17,35]$, the impact of peripheral LPS challenge on kynurenine pathway enzyme expression and 


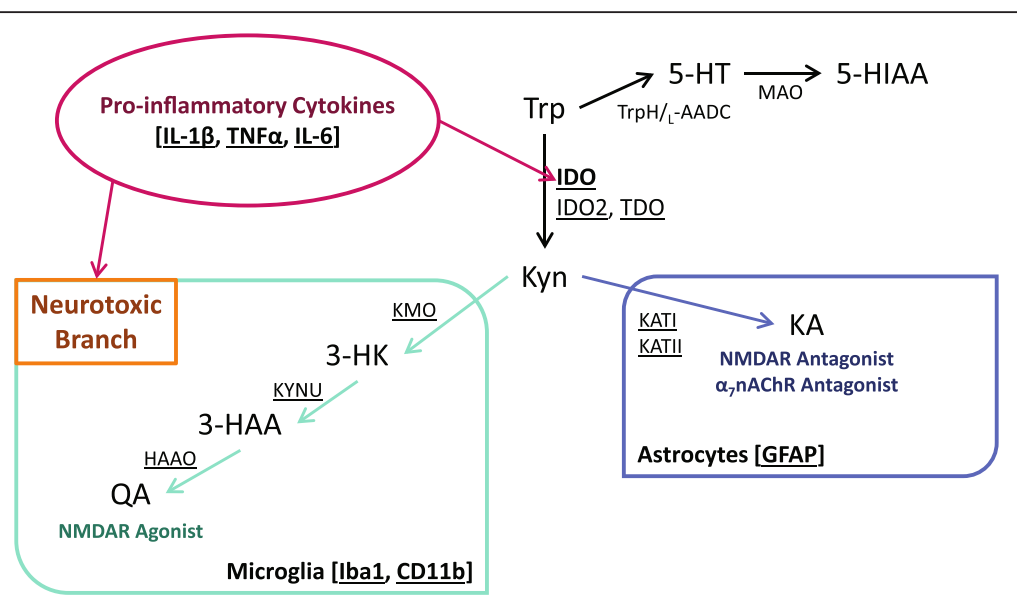

Fig. 1 Kynurenine pathway of tryptophan metabolism. Tryptophan is metabolized to kynurenine by indoleamine 2,3-dioxygenase (IDO), IDO-like enzyme, IDO2, and tryptophan 2,3-dioxygenase (TDO). Tryptophan can also be metabolized by tryptophan hydroxylase (TrpH) and L-aromatic amino acid decarboxylase ( $\_-A A D C$ ) to serotonin (5-HT) which is further broken down by monoamine oxidase (MAO) to 5-hydroxyindoleacetic acid (5-HIAA). Kynurenine can be metabolized to kynurenic acid (KA) by one of four isoforms of kynurenine aminotransferase (KAT), of which KATI and KATIl are most relevant to mammals. The production of KA by KATs mainly occurs in astrocytes, cells which predominantly express glial fibrillary acidic protein (GFAP). Alternately, kynurenine can be metabolized to 3-hydroxykynurenine (3-HK) by kynurenine 3-monooxygenase (KMO). 3-HK is metabolized to 3-hydroxyanthranilic acid (3-HAA) by kynureninase (KYNU) and 3-HAA to quinolinic acid (QA) by 3-hydroxyanthranilic acid dioxygenase (HAAO). This branch of the kynurenine pathway is compartmentalized in microglia, indicated by ionized calcium-binding adapter molecule 1 (Iba 1$)$ and CD11b expression. The elevation in expression of pro-inflammatory cytokines, such as interleukin-1 $\beta$ (IL-1 $\beta$ ), tumor necrosis factor a (TNFa), and interleukin-6 (IL-6), during inflammatory conditions activates glial cells. Further, IDO expression is up-regulated and flux through the neurotoxic kynurenine metabolic branch increases. Underlined targets were assessed by real-time RT-PCR to determine changes in mRNA

subsequent metabolism has only been described at the whole brain level [23]. Here, we tested the hypothesis that up-regulation of kynurenine pathway enzymes and disruption of kynurenine metabolic balance occurs in a regionally distinct manner. Brain regions associated with depressivelike behaviors were collected following peripheral immune challenge with LPS in mice. The steady-state mRNA expression of markers of glial activation, pro-inflammatory cytokines, and kynurenine pathway enzymes were measured. Tryptophan and its metabolites were also measured in each brain region. The brain regions assessed included the hippocampus, the amygdala, and the ventral striatum (nucleus accumbens). Dysfunction in these brain regions has been reported in depressed patients and is hypothesized to be a critical pathogenic mechanism underlying distinct depression-associated behaviors [36]. Because the periphery is reported to provide much of the brain's kynurenine [37], plasma metabolite levels were also measured. The data demonstrate that peripheral LPS treatment elevates the expression of pro-inflammatory cytokines and glial cell markers similarly in each region. IDO expression, but not IDO2 or tryptophan 2,3-dioxygenase (TDO), was significantly up-regulated after LPS treatment. Consistent with IDO up-regulation, the kynurenine/tryptophan ratio was elevated in each region; however, the 3hydroxykynurenine (3-HK)/KA ratio, an indicator of a neurotoxic imbalance in kynurenine metabolism, was only increased in the hippocampus (dorsal and ventral). These data are the first that characterize the brain region-specific metabolic response of the kynurenine pathway following peripheral LPS challenge.

\section{Methods}

\section{Animals}

All animal care and use was conducted in accord with the Guide for the Care and Use of Laboratory Animals, eighth edition (NRC), and protocols were approved by the Institutional Animal Care and Use Committee at the University of Texas Health Science Center at San Antonio (UTHSCSA). Male C57BL/6J mice were obtained from Jackson Laboratory (Bar Harbor, ME; stock\# 000664) and were used at ages between 8 and 12 weeks of age (adults). Prior to use, mice were group housed in standard shoebox cages, allowed ad libitum food and water access, and general health was monitored daily by veterinary technicians or research staff.

\section{Treatment}

Lipopolysaccharide (Sigma, St. Louis, MO) isolated from Escherichia coli (L-3129, serotype 0127:B8) was prepared fresh on the morning of injections and was dissolved in sterile, endotoxin-free $0.9 \%$ saline (vehicle) and injected intraperitoneally (i.p.) at a dose of $0.5 \mathrm{mg} / \mathrm{kg}$.

\section{Tissue sample collection}

At either 6 or 24 h following saline or LPS injections, mice were euthanized by carbon-dioxide asphyxiation. To measure mRNA expression of target genes, brain regions (amygdala, hippocampus, striatum) were grossly 
dissected (based on stereological coordinates in a mouse brain atlas [38]). Subsequent analysis of brain region metabolites was performed on microdissected brain regions harvested from serial 1-mm coronal brain sections using a brain matrix (Stoelting Co., Wood Dale, IL) (based on stereological coordinates in a mouse brain atlas [38]). Tissue was snap frozen in liquid nitrogen and stored at $-80{ }^{\circ} \mathrm{C}$ until analyzed. At $24 \mathrm{~h}$ following injections, prior to perfusion, blood was also collected for separation of plasma, which was stored at $-80{ }^{\circ} \mathrm{C}$.

\section{RNA isolation and real-time RT-PCR}

Brain tissues (hippocampus, amygdala, striatum) collected at 6 and $24 \mathrm{~h}$ post treatment (saline, LPS) were analyzed to determine relative steady-state mRNA expression using real-time RT-PCR. Isolation of RNA was carried out according manufacturer instructions using a PureLink ${ }^{\bullet}$ RNA Mini Kit (Life Technologies, Grand Island, NY), and cDNA was created using a high-capacity cDNA RT kit (Life Technologies) following manufacturer instructions. Real-time RT-PCR was performed over 40 cycles using a CFX384 ${ }^{\text {тм }}$ Real-Time PCR Detection System (Bio-Rad, Hercules, CA) and Taqman ${ }^{\bullet}$ Gene Expression Assays (Life Technologies): Gapdh (GAPDH, Mm99999915_g1), Il1b (IL-1ß, Mm013 36189_m1), Tnf (TNFa, Mm00443258_m1), Il6 (IL-6, Mm0 0446190_m1), Itgam (CD11b, Mm00434455_m1), Aif1 (Iba1, Mm00479862_g1), Gfap (GFAP, Mm01253033_m1), Ido (IDO, Mm00492586_m1), Ido2 (IDO2, Mm0052420 6_m1), Tdo2 (TDO, Mm00451266_m1), Ccbl1 (KATI, Mm01327703_m1), Aadat (KATII, Mm00496169_m1), Kmo (KMO, Mm00505511_m1), Kynu (KYNU, Mm00 551012_m1), and Haao (HAAO, Mm00517945_m1). Data are expressed as relative fold change (Target $\Delta_{\text {mRNA }}$ ) using the $2^{-\Delta \Delta C t}$ calculation method and GAPDH as the housekeeping gene as previously described [12].

\section{Liquid chromatography/mass spectrometry}

Microdissected brain regions (dorsal and ventral hippocampus, central amygdala, nucleus accumbens) and plasma were prepared for liquid chromatography/mass spectrometry (LC/MS) and analyzed for kynurenine metabolites as previously described [23]. Briefly, thawed plasma samples were diluted five times with $0.2 \%$ acetic acid and $1 \mathrm{mM}$ internal standards, transferred to Amicon Ultra filters (Millipore, Billerica, MA) and centrifuged at $13,500 \times g$ for $1 \mathrm{~h}$ at $4{ }^{\circ} \mathrm{C}$. Frozen brain regions were diluted 30 times with $0.2 \%$ acetic acid and $1 \mathrm{mM}$ internal standards and then homogenized at $4{ }^{\circ} \mathrm{C}$ using a Bead Ruptor 24 Homogenizer (Omni International, Kennesaw, GA) with 1.4-mm zirconium ceramic oxide beads (Omni International) and settings of (pulse duration $45 \mathrm{~s}$, pulse number 2, rest interval $15 \mathrm{~s})$. The supernatant was filtered in a centrifuge as the plasma. Following preparation, samples were analyzed on a Q Exactive mass spectrometer (Thermo Fisher Scientific,
Waltham, MA) with on-line separation by a Dionex UltiMate 3000 HPLC system (Thermo Fisher Scientific), and the data collected was analyzed using Xcalibur 2.2 software (Thermo Fisher Scientific) in the Mass Spectrometry Core Facility at the University of Texas Health Science Center at San Antonio. Metabolite ratios were calculated, as an in vivo estimation of IDO activity, kynurenine metabolic balance, and serotonin turnover, from raw data that are part of a separate study (Parrott JM, Redus L, Morales J, Xiaoli G, O'Connor JC: Neurotoxic kynurenine metabolism is increased in the dorsal hippocampus and drives distinct depressive behaviors during inflammation, Submitted). These values were used to determine the following ratios: kynurenine/tryptophan, 3-HK/KA, and 5-HIAA/5-HT. 3-Hydroxyanthranilic acid (3-HAA) and QA were not reliably detected in brain region microdissected samples due to limited sample size.

\section{Statistical analysis}

Data were analyzed using SigmaPlot 12.0 software (Systat Software Inc., San Jose, CA) and are presented as group means + standard error of the mean (SEM). Following a single-pass Chauvenet's test for outliers as previously described [26], data analysis was conducted using either a $t$ test or a one-way analysis of variance (ANOVA). Significant main effects identified by the ANOVA were further analyzed with the Holm-Sidak method for pairwise multiple comparisons post hoc test to identify between-group differences. Significant treatment effects $(p<0.05$, compared to saline) are denoted as such $(*)$, and trends $(p<0.1-0.05)$ are denoted as such $\left({ }^{\#}\right)\left({ }^{\#} p<0.1-0.05 ; * p<0.05-0.01\right.$; *** $p<0.01-0.001 ; * * * 0<0.001)$.

\section{Results}

Peripheral lipopolysaccharide induces time-dependent central pro-inflammatory cytokine expression

Inflammation precipitates depressive-like behaviors that are controlled by neurocircuits within distinct regions of the brain, yet the impact of peripheral immune activation on pro-inflammatory cytokine expression within these regions has not been thoroughly characterized. To confirm the time course and regional response of pro-inflammatory cytokine expression following peripheral LPS challenge, interleukin$1 \beta$ (IL-1 $\beta$ ), tumor necrosis factor $\alpha$ (TNF $\alpha$ ), and interleukin-6 (IL-6) were measured 6 and $24 \mathrm{~h}$ post treatment. In the hippocampus, IL-1 $\beta$ expression (Fig. 2a, left panel) was elevated relative to saline at both $6 \mathrm{~h}(p<0.001)$ and $24 \mathrm{~h}(p<0.001)$ post LPS injections. Similarly, TNF $\alpha$ expression (Fig. 2a, center panel) also increased from saline following LPS injections at both $6 \mathrm{~h}(p<0.001)$ and $24 \mathrm{~h}$ $(p<0.001)$. IL-6 expression (Fig. 2a, right panel) was only elevated at $6 \mathrm{~h}(p<0.001)$ post LPS treatment and returned to saline expression levels by $24 \mathrm{~h}$. In the amygdala, both IL-1 $\beta$ (Fig. 2b, left panel) and TNF $\alpha$ expression (Fig. 2b, 


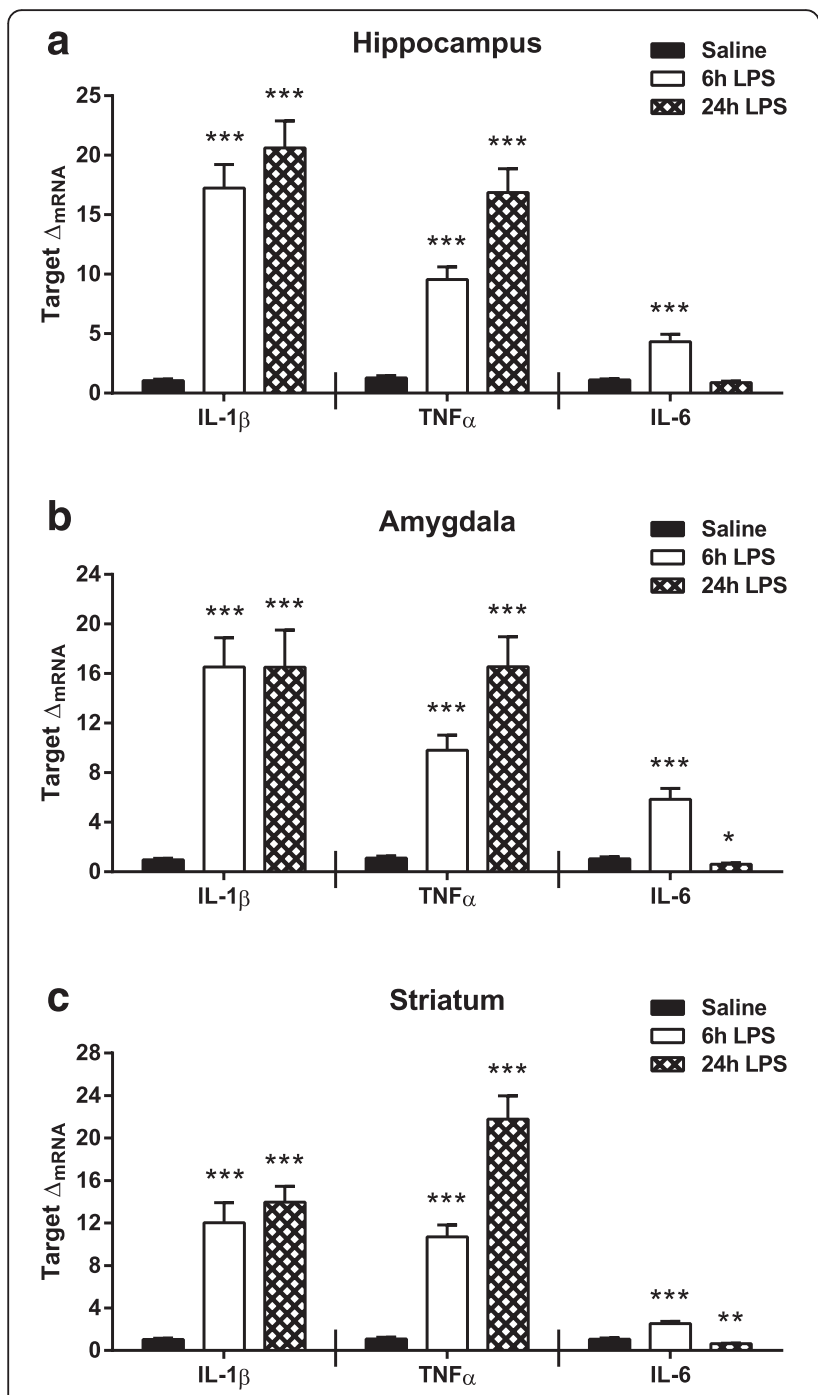

Fig. 2 Central pro-inflammatory cytokines are elevated following peripheral LPS injections. mRNA fold changes $\left(\Delta_{\text {mRNA }}\right)$ of interleukin-1 $\beta$ (IL-1 $\beta$, left panel), tumor necrosis factor a (TNFa, middle panel), and interleukin-6 (IL-6, right panel) were measured at 6 and $24 \mathrm{~h}$ post LPS treatment in the (a) hippocampus, (b) amygdala, and (c) striatum. Data represent sample means \pm SEM, $n=8-11$ samples/group. ${ }^{*}=$ post hoc comparison to saline treatment group. ${ }^{*} p<0.05-0.01 ;{ }^{* *} p<0.01-0.001 ;{ }^{* * *} p<0.001$

center panel) followed a similar pattern of increased expression at $6 \mathrm{~h}(p<0.001 \mathrm{IL}-1 \beta ; p<0.001 \mathrm{TNF} \alpha)$ and $24 \mathrm{~h}$ $(p<0.001 \mathrm{IL}-1 \beta ; p<0.001 \mathrm{TNF} \alpha)$ following LPS injections. As in the hippocampus, IL-6 expression (Fig. 2b, right panel) in the amygdala was elevated at $6 \mathrm{~h}(p<0.001)$ post LPS treatment; however, expression was significantly decreased relative to saline at $24 \mathrm{~h}(p=0.018)$ post-LPS. IL-1 $\beta$ (Fig. 2c, left panel) and TNF $\alpha$ expression (Fig. 2c, center panel) in the striatum was elevated compared to saline at both $6 \mathrm{~h}(p<0.001 \mathrm{IL}-1 \beta ; p<0.001$ TNF $\alpha)$ and $24 \mathrm{~h}(p<0.001$ IL-1 $\beta ; p<0.001$ TNF $\alpha)$ post LPS injections. IL-6 expression (Fig. 2c, right panel) in the striatum only increased at $6 \mathrm{~h}(p<0.001)$ following LPS treatment and decreased relative to saline levels by $24 \mathrm{~h}(p$ $=0.001$ ) post injections. Together, these data demonstrate that in the hippocampus, amygdala, and striatum, there is a similar pro-inflammatory cytokine temporal response following a peripheral inflammatory stimulus, such as LPS.

\section{Markers of glial activation increase in response to peripheral LPS challenge}

Previous studies have demonstrated regional heterogeneity in microglia cell distribution [39] and activation following peripheral LPS injections [40]. To determine the temporal and regional expression of glial markers following peripheral LPS, mRNA expression of cluster of differentiation molecule 11b (CD11b), ionized calciumbinding adapter molecule 1 (Iba1), and glial fibrillary acidic protein (GFAP) were measured. In the hippocampus, CD11b expression (Fig. 3a, left panel), a cellular marker of microglia, was significantly elevated at $24 \mathrm{~h}$ $(p<0.001)$ post LPS treatment. Iba1 (Fig. 3a, center panel), another marker of microglia activation, was lower than saline at $6 \mathrm{~h}(p<0.001)$ while expression was significantly elevated at $24 \mathrm{~h}(p<0.001)$ post LPS injections. The astrocytic marker, GFAP (Fig. 3a, right panel), was also up-regulated relative to saline at both $6 \mathrm{~h}(p<0.001)$ and $24 \mathrm{~h}(p<0.001)$ following peripheral LPS treatment. In the amygdala (Fig. 3b, left, center, right panels), the expression pattern of glial markers was nearly identical to that observed in the hippocampus, with the exception that post hoc analysis revealed that CD11b expression was not significantly different from saline at either 6 or $24 \mathrm{~h}$ post LPS. In the striatum, CD11b expression (Fig. 3c, left panel) decreased at $6 \mathrm{~h}(p=0.021)$ and increased at $24 \mathrm{~h}(p=0.043)$ following LPS injections. Iba1 expression (Fig. 3c, center panel) in the striatum decreased at $6 \mathrm{~h}(p<0.001)$ and was elevated at $24 \mathrm{~h}(p<0.001)$ post LPS treatment. Finally, GFAP expression (Fig. 3c, right panel) was up-regulated relative to saline expression at both $6 \mathrm{~h}(p<0.001)$ and $24 \mathrm{~h}(p<0.001)$ after peripheral LPS. Similar to pro-inflammatory cytokines, these data suggest that glial activation is relatively uniform across discrete brain regions with up-regulation of astrocytic markers preceding microglial markers.

\section{Peripheral LPS challenge increases central indoleamine 2,3-dioxygenase expression}

Previous studies have established that pro-inflammatory cytokines up-regulate the expression of IDO which is necessary for the development of inflammation-associated depressive-like behaviors [19]. However, IDO2 and TDO are also expressed in the brain and whether they contribute to increased kynurenine metabolism after peripheral LPS challenge is not known. Therefore, the expression of IDO, TDO, and IDO2 (Fig. 1) was assessed. IDO expression 


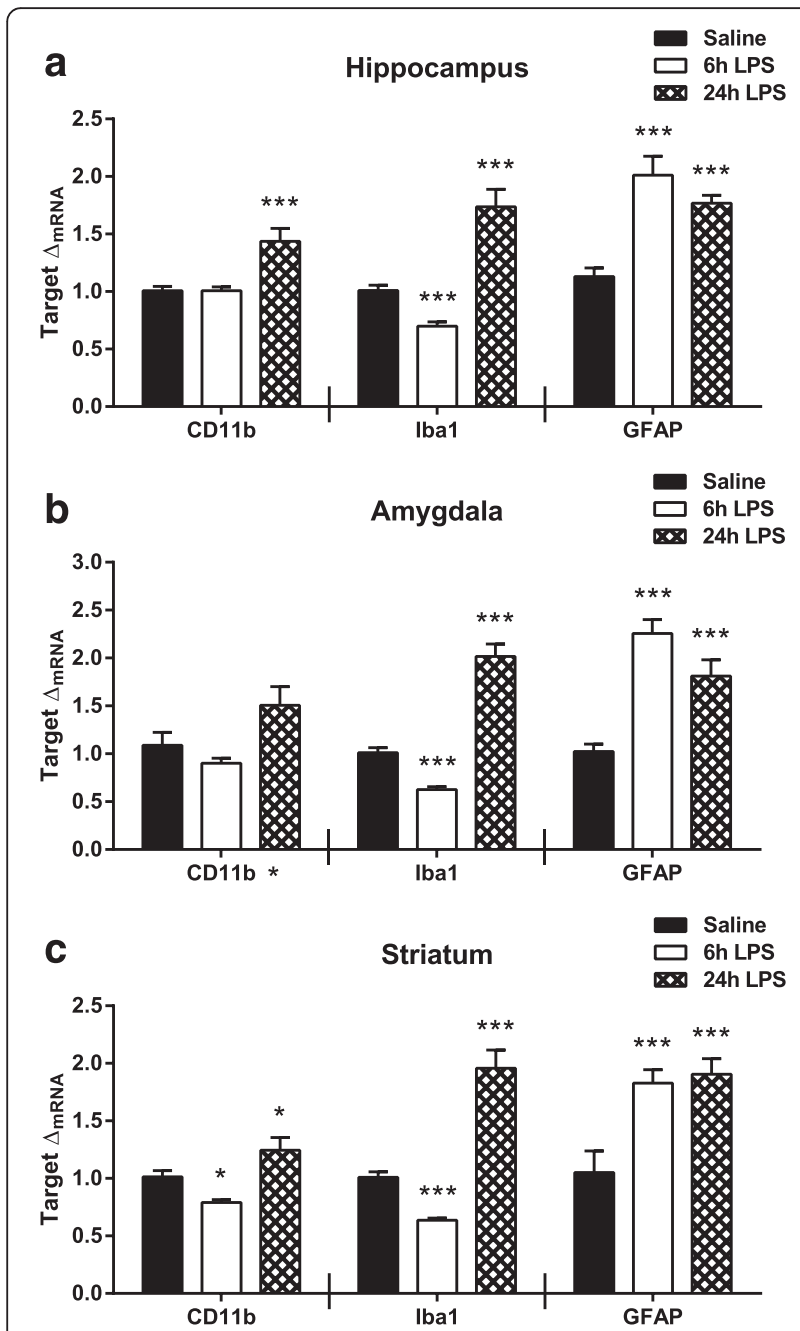

Fig. 3 Peripheral inflammation up-regulates central expression of glial cell markers. mRNA fold changes $\left(\Delta_{\mathrm{mRNA}}\right)$ of CD11b (left panel), ionized calcium-binding adapter molecule 1 (Ibal, middle panel), and glial fibrillary acid protein (GFAP, right panel) were measured at 6 and $24 \mathrm{~h}$ post LPS treatment in the $\mathbf{a}$ hippocampus, $\mathbf{b}$ amygdala, and $\mathbf{c}$ striatum. Data represent sample means \pm SEM, $n=9-11$ samples/ group. ${ }^{*}=$ overall significance or post hoc comparison to saline treatment group. ${ }^{*} p<0.05-0.01 ;{ }^{* *} p<0.01-0.001 ;{ }^{* *} p<0.001$

(Fig. $4 \mathrm{a}-\mathrm{c}$ ) was elevated relative to saline at both $6 \mathrm{~h}$ $(p<0.001$ hippocampus, $p<0.001$ amygdala, $p<0.001$ striatum) and $24 \mathrm{~h}(p<0.001$ hippocampus, $p<0.001$ amygdala, $p<0.001$ striatum) post LPS treatment. In the hippocampus (Fig. 4d, left panel) and amygdala (Fig. 4e, left panel), IDO-like enzyme (IDO2) expression was decreased from saline expression at $24 \mathrm{~h}(p<0.001$ hippocampus, $p$ $=0.042$ amygdala) following LPS administration, while no significant difference was observed in the striatum. There was a trend for an overall effect of time for TDO expression $\left(F_{2,24}=3.12, p=0.063\right)$ in the hippocampus (Fig. $4 \mathrm{~d}$, right panel). In the amygdala, there was not a significant change in TDO expression (Fig. 4e, right panel) following LPS treatment. Similar to the hippocampus, striatal TDO expression (Fig. 4e, right panel) tended toward an overall effect of time $\left(F_{2,27}=2.95, p=0.069\right)$. Together, these data demonstrate that IDO is the only rate-limiting enzyme of the kynurenine pathway that is significantly up-regulated following peripheral immune challenge with LPS.

\section{Kynurenine aminotransferases I and II are impacted in a regionally dependent manner following peripheral lipopolysaccharide}

Under basal conditions, the majority of kynurenine is metabolized to KA by kynurenine aminotransferases (KAT) I and II (Fig. 1), the expression of which is not thought to be impacted by inflammation [41]. The regional and temporal mRNA expression pattern of KATI and KATII was measured. In the hippocampus, KATI expression (Fig. 5a, left panel) was significantly elevated at $6 \mathrm{~h}(p=0.005)$ and significantly decreased at $24 \mathrm{~h}$ $(p=0.001)$ relative to saline following LPS treatment. KATII expression in the hippocampus (Fig. 5a, right panel) was significantly decreased at $24 \mathrm{~h}(p=0.013)$ following treatment with LPS. In the amygdala, both KATI (Fig. 5b, left panel, $F_{2,27}=3.35, p=0.050$ ) and KATII expression (Fig. 5b, right panel, $F_{2,27}=2.93, p=$ 0.071 ) trended toward significance for an overall effect of time following LPS injections. In the striatum, there was a significant overall effect of time for KATI expression (Fig. 5c, left panel, $F_{2,26}=5.32, p=0.012$ ) after LPS treatment, though neither 6 -h expression nor 24-h expression was significantly different from saline. Similarly, striatal KATII expression (Fig. 5b, right panel, $F_{2,24}=7.78, p=0.002$ ) was significantly impacted by LPS in which 6-h expression $(p=0.096)$ trended toward a decrease while 24-h expression $(p=0.074)$ trended toward an increase relative to saline expression. These data suggest that peripheral LPS treatment does cause a modest time- and region-dependent change in the expression of KATI and KATII within the brain.

\section{Peripheral LPS induces expression changes in neurotoxic kynurenine pathway enzymes}

Though it has been demonstrated that peripheral LPS increases whole brain metabolism of kynurenine through the neurotoxic branch [23], the regional or temporal expression pattern of the associated pathway enzymes has not been reported. Kynurenine 3-monooxygenase (KMO), kynureninase (KYNU), and 3-hydroxyanthranilic acid dioxygenase (HAAO) (Fig. 1) mRNA was measured by real-time RT-PCR. In the hippocampus, KMO expression (Fig. 6a, left panel) was significantly elevated at $6 \mathrm{~h}$ ( $p=0.024)$ following LPS treatment. KYNU expression (Fig. 6a, middle panel) was unchanged in response to peripheral LPS. Hippocampal HAAO expression (Fig. 6a, right panel) significantly decreased at $6 \mathrm{~h}$ post LPS treatment $(p<0.001)$ and returned to saline expression levels 

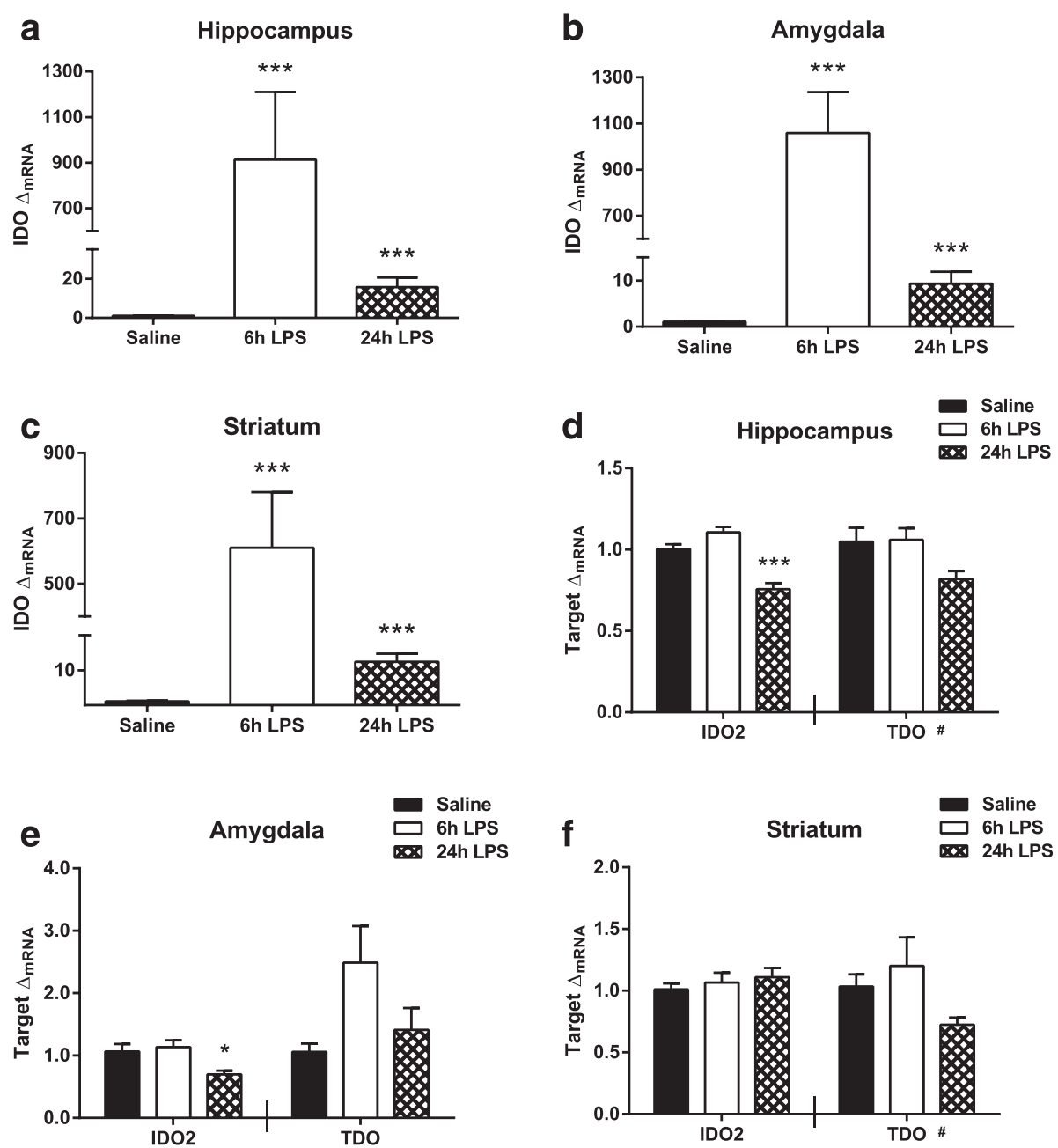

Fig. 4 Peripheral LPS specifically increases expression of kynurenine producing enzyme, indoleamine 2,3-dioxygenase (IDO). mRNA fold changes $\left(\Delta_{\text {mRNA }}\right)$ of IDO were assessed at 6 and $24 \mathrm{~h}$ following LPS injections in the (a) hippocampus, (b) amygdala, and (c) striatum. mRNA expression of IDO2 (left panel) and tryptophan 2,3-dioygenase (TDO, right panel) were also measured at 6 and $24 \mathrm{~h}$ post LPS in the (d) hippocampus, (e) amygdala, and (f) striatum. Data represent sample means \pm SEM, $n=8-11$ samples/group. $,{ }^{*},=$ overall significance or post hoc comparison to saline treatment group. ${ }^{\#} p<0.1-0.05 ;{ }^{*} p<0.05-0.01 ;{ }^{* *} p<0.01-0.001 ;{ }^{* * *} p<0.001$

by $24 \mathrm{~h}$. The same expression pattern was measured in the amygdala for each target. KMO mRNA was significantly increased (Fig. 6b, left panel, $p<0.001$ ), KYNU was unchanged (Fig. 6b, middle panel), and HAAO expression (Fig. 6b, right panel) was decreased at $6 \mathrm{~h}(p=0.004)$ but not $24 \mathrm{~h}$ following LPS treatment. Finally, in the striatum, KMO expression (Fig. 6c, left panel) was significantly elevated at $6 \mathrm{~h}(p=0.015)$ and returned to saline expression at $24 \mathrm{~h}$ post LPS. A significant overall effect of time on striatal KYNU expression (Fig. 6c, middle panel) was apparent following LPS $\left(F_{2,25}=3.44, p=0.048\right)$; however, post hoc testing revealed that 6 -h expression $(p=0.087)$ and 24-h expression $(p=0.074)$ only trended to decrease from saline expression. HAAO expression (Fig. 6c, right panel) also decreased at $6 \mathrm{~h}(p<0.001)$ and returned to saline levels $24 \mathrm{~h}$ following LPS treatment. Taken together, these data indicate that peripheral immune challenge with LPS causes a robust increase in the expression of KMO mRNA with a concomitant time and region-dependent down-regulation of KYNU and HAAO.

\section{Peripheral LPS causes a neurotoxic shift in hippocampal kynurenine metabolism}

Multiple studies have described the impact of LPS on central tryptophan metabolism [19, 23, 42, 43], but little is known about region-dependent tryptophan metabolism during peripheral inflammation. The kynurenine/ tryptophan ratio, 3-HK/KA ratio, and 5-HIAA/5-HT ratio were determined in four brain regions $24 \mathrm{~h}$ post LPS treatment. LPS induced a significant increase in the kynurenine/tryptophan ratio in each brain region: dorsal hippocampus (Fig. 7a, $p<0.001$ ), ventral hippocampus 


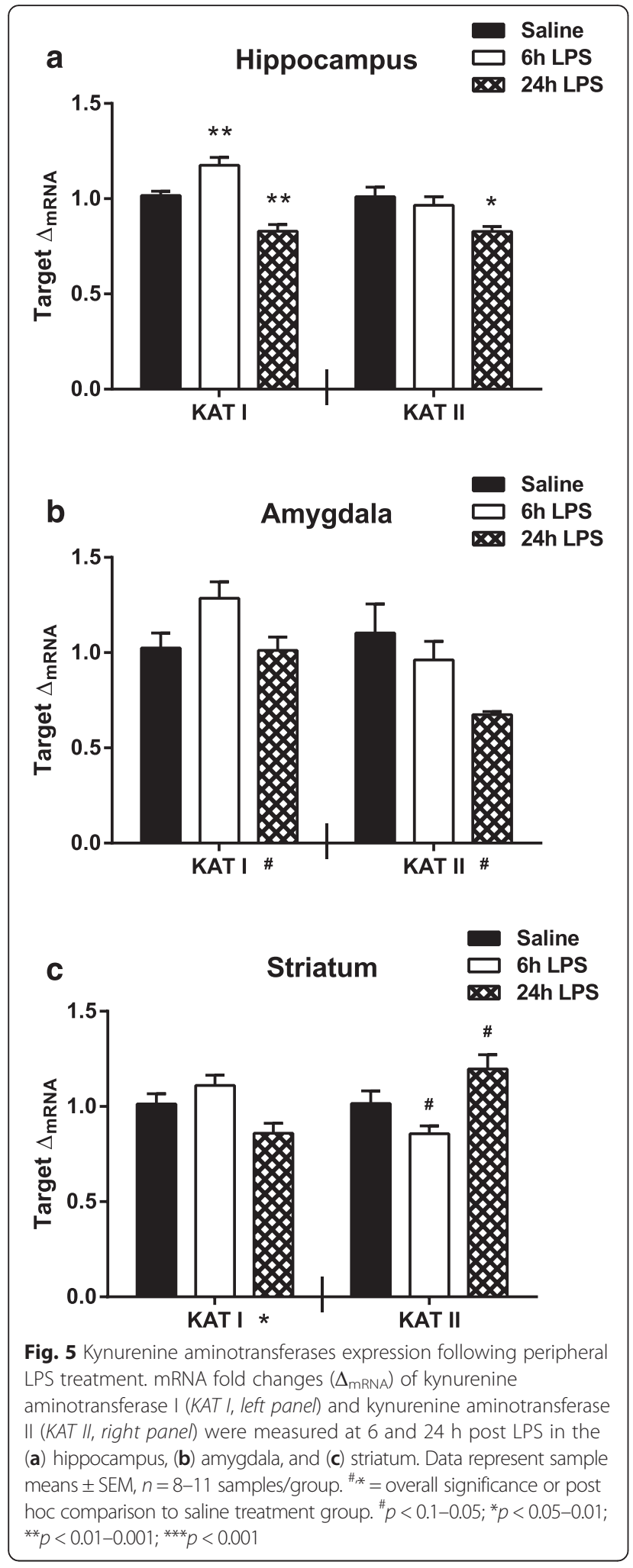

(Fig. 7b, $p<0.001$ ), central amygdala (Fig. 7c, $p<0.001$ ), and nucleus accumbens (Fig. $7 \mathrm{~d}, p<0.001$ ). The balance of downstream kynurenine metabolism was shifted toward the neurotoxic branch in the hippocampus with

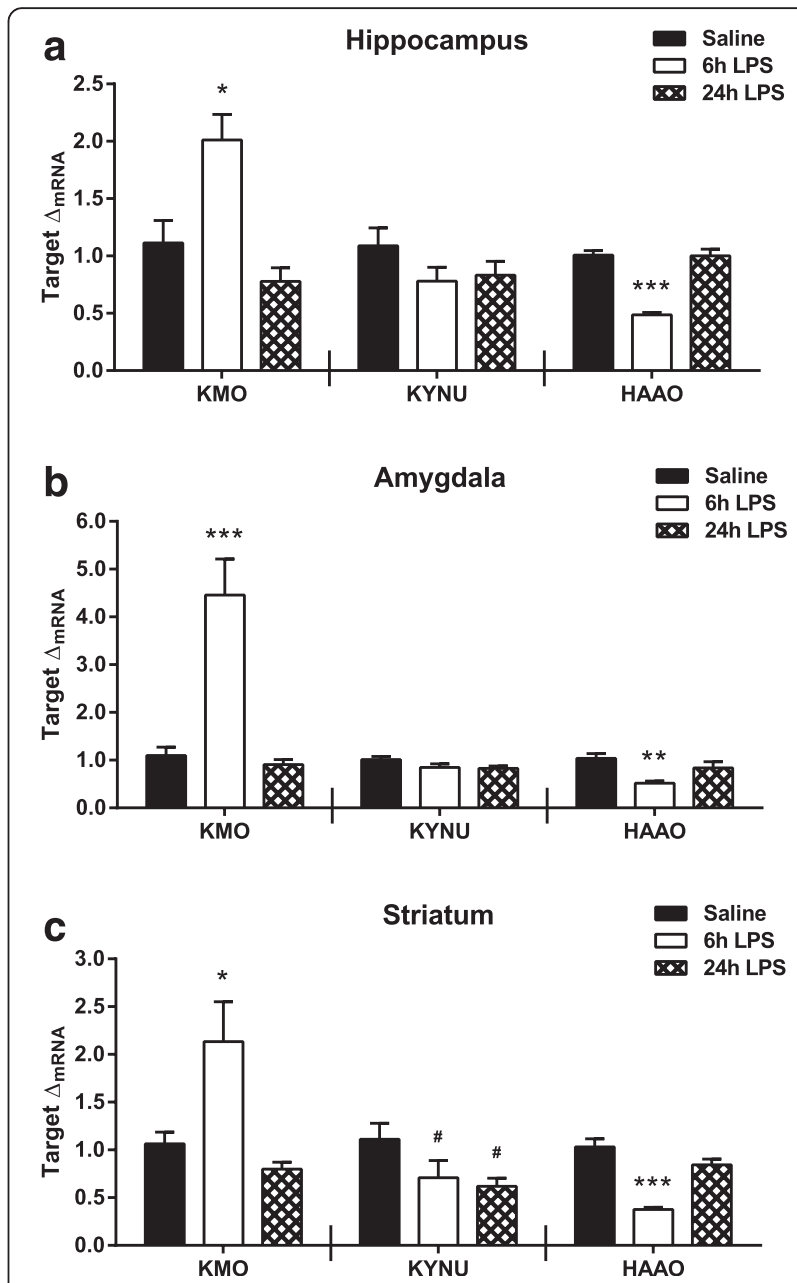

Fig. 6 Peripheral inflammation alters expression of downstream kynurenine pathway enzymes. mRNA fold changes $\left(\Delta_{\mathrm{mRNA}}\right)$ of kynurenine 3-monooxygenase (KMO, left panel), kynureninase (KYNU, middle panel), and 3-hydroxyanthranilic acid dioxygenase (HAAO, right panel) were measured at 6 and $24 \mathrm{~h}$ following LPS injections in the (a) hippocampus, (b) amygdala, and (c) striatum. Data represent sample means \pm SEM, $n=9-11$ samples/group. ${ }^{\# * *}=$ post hoc comparison to saline treatment group. ${ }^{*} p<0.1-0.05$; ${ }^{*} p<0.05-0.01$; ${ }^{* *} p<0.01-0.001$; ${ }^{* * *} p<0.001$

the 3-HK/KA ratio significantly increased (dorsal; Fig. 7e, left panel, $p=0.054$ and ventral; Fig. 7f, left panel, $p=$ 0.032). The $3 \mathrm{HK} / \mathrm{KA}$ ratio was not different $24 \mathrm{~h}$ post LPS in central amygdala (Fig. $7 \mathrm{~g}$, left panel) or nucleus accumbens (Fig. 7h, left panel). While serotonin levels were unchanged (data not shown), 5-HT metabolism to 5 -HIAA was increased $24 \mathrm{~h}$ post LPS in the dorsal hippocampus (Fig. 7e, right panel, $p<0.001$ ), ventral hippocampus (Fig. 7f, right panel, $p<0.001$ ), and central amygdala (Fig. 7g, right panel, $p=0.071$ ). While the nucleus accumbens 5-HIAA/5-HT ratio was not significantly increased (Fig. 7h, right panel). Together, these data demonstrate that LPS causes a general brain region-independent increase in tryptophan metabolism and serotonin turnover, 


\section{a}

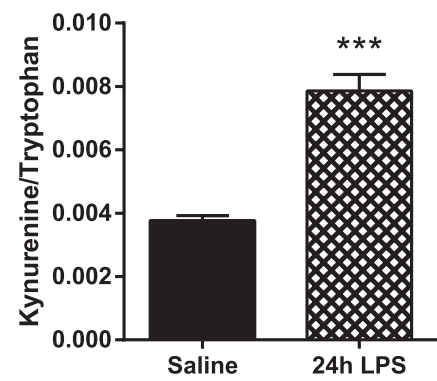

C

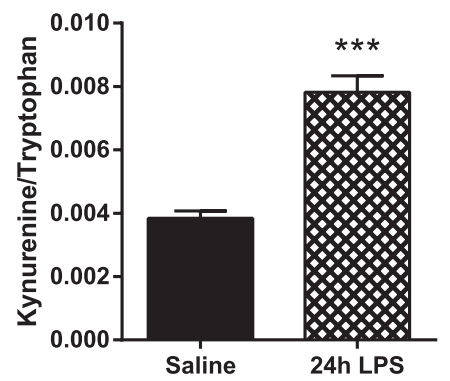

e

Dorsal

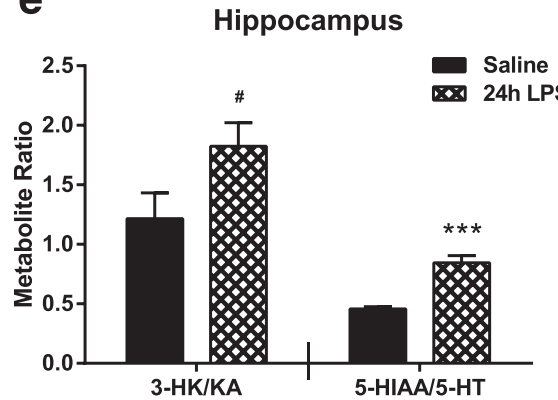

g

Central Amygdala

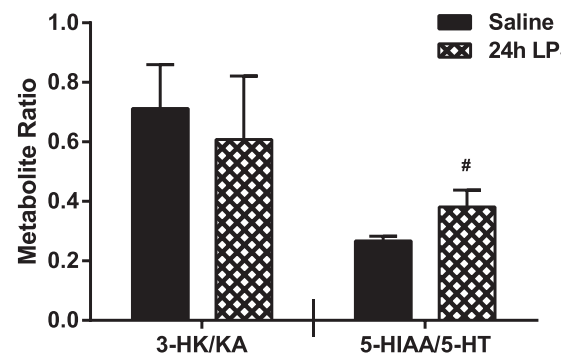

b Ventral Hippocampus

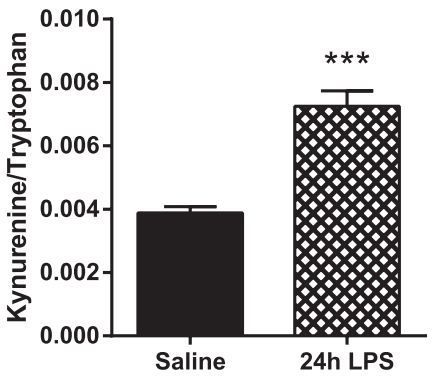

d Nucleus Accumbens

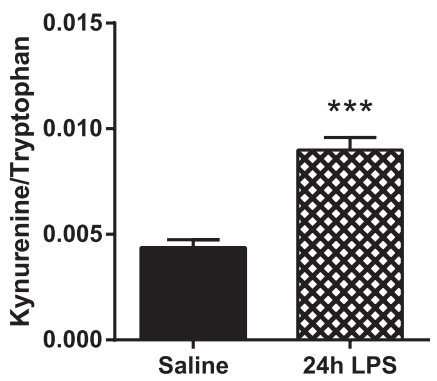

f

Ventral

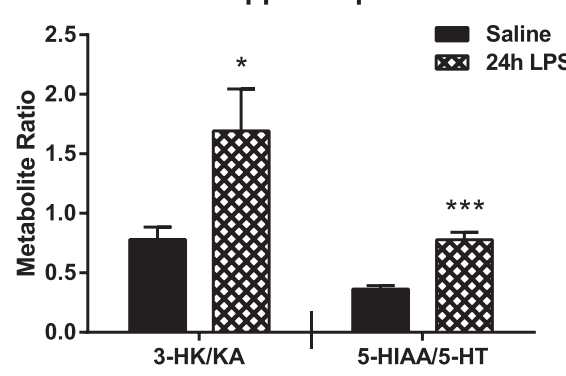

h Nucleus

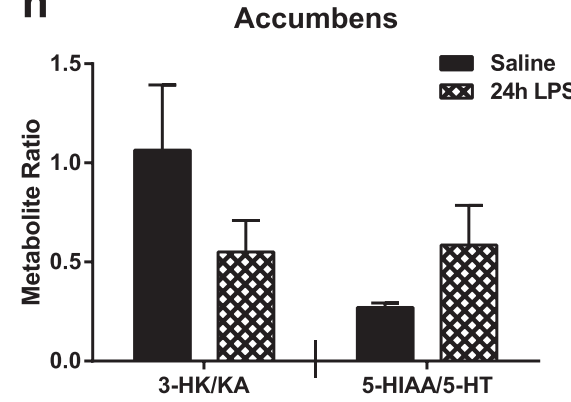

Fig. 7 Kynurenine/tryptophan ratio and hippocampal neurotoxic kynurenine metabolite ratio are up-regulated in response to peripheral LPS. The kynurenine/tryptophan ratio was measured at $24 \mathrm{~h}$ following LPS injections in the (a) dorsal hippocampus, (b) ventral hippocampus, (c) central amygdala, and (d) nucleus accumbens. Metabolite ratios of 3-hydroxykynurenine/kynurenic acid (3-HK/KA, neurotoxic ratio, left panel) and 5-hydroxyindoleacetic acid/serotonin (5-HIAA/5-HT, serotonin turnover, right panel) were measured $24 \mathrm{~h}$ post LPS in the (e) dorsal hippocampus, (f) ventral hippocampus, (g) central amygdala, and (h) nucleus accumbens. Data represent sample means \pm SEM, $n=7-12$ samples/group. ${ }^{\#} p<0.1-0.05 ;{ }^{*} p<0.05-0.01 ;{ }^{* *} p<0.01-0.001 ;{ }^{* * *} p<0.001$ 
but downstream neuroactive kynurenine metabolism occurs in a region-dependent manner.

\section{Lipopolysaccharide increases peripheral kynurenine metabolism toward the neurotoxic route}

Because peripheral circulation is a significant source of kynurenine for the brain [37], plasma tryptophan metabolism was measured (Table 1). LPS treatment significantly increased the concentration of tryptophan (Table 1, $p=0.006$ ), kynurenine (Table $1, p<0.001$ ), and 3 -HK (Table 1, $p<0.001)$. The concentration of 3-HAA (Table 1), QA (Table 1), and KA (Table 1) remained unchanged $24 \mathrm{~h}$ after LPS treatment. The kynurenine/tryptophan ratio (Table $1, p<0.001$ ) and the 3 -HK/KA ratio (Table $1, p<0.001$ ) were also significantly increased following LPS injections. These data indicate that LPS precipitates a robust up-regulation of kynurenine metabolism that is shifted toward the neurotoxic branch; however, neuroactive metabolic end products were not increased.

\section{Discussion}

Under the guidelines of the $\mathrm{RDoC}$ framework, a renewed emphasis has been placed on understanding regionally distinct structural, cellular, and molecular events involved in the pathogenesis of neuropsychiatric disease $[29,30]$. Therefore, the objective of this study was to characterize the LPS-induced neuroinflammatory response in brain regions relevant to depression-related symptoms as previous literature has described these data only at the whole brain level. Up-regulation of proinflammatory cytokine expression (IL-1 $\beta$, TNF $\alpha$, and IL-6) followed the same temporal pattern in each of the three regions studied (hippocampus, amygdala, and striatum) following peripheral LPS challenge. The relative magnitude of the LPS-induced cytokine response was similar as well. Likewise, glial cell markers (CD11b, Iba1, and GFAP), in general, were elevated in a region-independent manner. Interestingly, up-regulation of astrocytic marker GFAP preceded the subsequent increase in microglial markers $\mathrm{CD} 11 \mathrm{~b}$ and Iba1. Consistent with studies demonstrating that IDO expression is induced by pro-inflammatory cytokines, IDO expression was robustly up-regulated in a region-independent manner, peaking at $6 \mathrm{~h}$ following LPS treatment, while IDO2 and TDO remained largely unchanged. Interestingly, the expression of neurotoxic kynurenine pathway enzymes downstream of IDO (KMO, KYNU, and HAAO) was also similar between brain regions; however, each enzyme had a unique response pattern following peripheral inflammation. KMO expression increased, KYNU expression remained unaffected, and HAAO expression decreased as an immediate response to LPS treatment. On the opposing metabolic branch, KAT enzymes (I and II) were significantly down-regulated in the hippocampus, $24 \mathrm{~h}$ after LPS treatment. In accord with the mRNA data, functional assessment of kynurenine metabolism showed that the kynurenine/tryptophan ratio increased approximately twofold following peripheral immune activation in the same pattern throughout the regions assessed. Interestingly, the 3-HK/KA ratio, which reflects the relative neurotoxic/neuroprotective metabolic balance, was only up-regulated in the hippocampal regions (dorsal and ventral) following LPS treatment. Together, these data demonstrate that peripheral immune challenge results in region-independent induction of neuroinflammation and kynurenine production, while downstream metabolism of kynurenine is region specific.

To characterize the neuroinflammatory response associated with peripheral immune activation, pro-inflammatory cytokine and glial cellular marker expressions were assessed. The expression of all three pro-inflammatory cytokines, IL-1 $\beta$, TNF $\alpha$, and IL- 6 were increased $6 \mathrm{~h}$ after LPS injections in all three brain regions, similar to previously published data from the hippocampus, cortex, and hypothalamus $[17,44]$. This early central proinflammatory response drives the development of LPSinduced sickness behavior (lethargy, decreased appetite,

Table 1 Peripheral inflammation increases flux through the kynurenine pathway of tryptophan metabolism

\begin{tabular}{|c|c|c|c|}
\hline \multirow[t]{2}{*}{ Metabolite } & \multicolumn{2}{|c|}{ Concentration ( $\mu \mathrm{M})$} & \multirow{2}{*}{$\begin{array}{l}t \text { test } \\
p \text { value }\end{array}$} \\
\hline & Saline & $24 \mathrm{~h} \mathrm{LPS}$ & \\
\hline Tryptophan & $94.93(7.1)$ & $119.53(3.9)$ & $p<0.01$ \\
\hline Kynurenine & $0.83(0.08)$ & $2.40(0.2)$ & $p<0.001$ \\
\hline 3-Hydroxykynurenine (3-HK) & $0.16(0.02)$ & $0.27(0.04)$ & $p<0.001$ \\
\hline 3-Hydroxyanthranilic acid (3-HAA) & $3.99(0.3)$ & $4.87(0.4)$ & n.s. \\
\hline Quinolinic acid (QA) & $0.98(0.06)$ & $0.97(0.07)$ & n.s. \\
\hline Kynurenic acid (KA) & $0.24(0.03)$ & $0.27(0.01)$ & n.s. \\
\hline Kynurenine/tryptophan ratio & $0.0092(0.0004)$ & $0.020(0.005)$ & $p<0.001$ \\
\hline 3-HK/KA ratio & $0.55(0.04)$ & $1.08(0.07)$ & $p<0.001$ \\
\hline
\end{tabular}

Kynurenine pathway metabolites $(\mu \mathrm{M})$ were assessed by LC/MS in the plasma $24 \mathrm{~h}$ following saline or LPS treatment. Metabolites measured include tryptophan, kynurenine, 3-hydroxykynurenine (3-HK), 3-hydroxyanthranilic acid (3-HAA), quinolinic acid (QA), and kynurenic acid (KA). Kynurenine/tryptophan and 3-HK/KA ratios were calculated. Data represent sample means (SEM) and were analyzed using a $t$ test (results denoted in table). $n=7-12$ samples/group, n.s. not significant 
reduced social interactions, fever) $[45,46]$. At $24 \mathrm{~h}$ post-LPS treatment, IL-1 $\beta$ and TNF $\alpha$ expression remained elevated while IL- 6 returned to saline expression levels, also an effect similar to previously published results [17]. It is important to note that under the experimental conditions of this study, $24 \mathrm{~h}$ after LPS treatment corresponds to when depressive-like behaviors have been assessed previously [47]. It is possible that the culmination of IL- 6 prior to IL- $1 \beta$ and TNF $\alpha$ expression is associated with the resolution of a specific behavior, such as fever [48], while other behaviors persist at $24 \mathrm{~h}$ post-LPS and IL- $1 \beta$ and TNF $\alpha$ play an important role in the development of those behaviors. It is important to note that using steady-state mRNA analysis of brain neuroinflammatory targets has both merit and limitations. In our study, mRNA targets allow for an extremely sensitive and quantitative measure of neuroinflammatory gene expression. Since many proinflammatory cytokines, including IL-1 $\beta$, TNF $\alpha$, and IL6 , are able to enter the brain via transport across the blood brain barrier $[49,50]$, determination of mRNA expression ensures that any changes being measured reflect the local brain expression of the targets, not the appearance/accumulation of peripherally secreted cytokines. However, it is possible that variability in translation dynamics of mRNA to protein could influence the expression of de novo synthesized neuroinflammatory targets.

Interestingly, the early up-regulation of central proinflammatory cytokine mRNA was coupled with a general down-regulation of microglial markers $(\mathrm{CD} 11 \mathrm{~b}$ and Iba1) and increase of astrocytic GFAP. At both time points after LPS, expression of astrocyte cell marker GFAP was up-regulated, which is consistent with a previous study demonstrating that central LPS administration increases GFAP expression [51]. Microglia are known to be the predominant cellular producers of proinflammatory cytokines in the brain, and Iba1 expression and/or staining are often reported as a proxy of activation state [52]. The incongruence in the expression patterns between the gene targets in our study suggests that expression of pro-inflammatory cytokines precede the up-regulation of microglia-specific markers. Additionally, utilization of CD11b or Iba1, in the absence of morphologic or other complementary data, appears to be an insufficient representation of microglia activation state. Moreover, the temporal difference between up-regulation of astrocyte versus microglial markers suggests the possibility of a dynamic role for these two cell types in mediating the neuroinflammatory response. This could be particularly relevant in terms of kynurenine metabolism which is a pathway compartmentalized between astrocytes and microglia in the brain $[24,53]$. It is interesting to note that both CD11b and GFAP are increased in their respective cell populations by increases in nitric oxide $(\mathrm{NO})$ production $[54,55]$, which can be generated by elevations in pro-inflammatory cytokines and kynurenine metabolite QA [56-58]. Further investigation is warranted to more precisely explore this phenomenon.

The expression of all known rate-limiting kynurenine producing enzymes, IDO, IDO2, and TDO, was assessed to better understand how tryptophan is metabolized following peripheral LPS. IDO expression was increased at both 6 and 24 h post LPS injections in each brain region assessed, consistent with two previously published studies $[17,44]$. IDO2 is a more recently discovered homologue of IDO with much lower enzymatic activity compared to IDO [59]. In the hippocampus and amygdala, IDO2 expression decreased at 24 h following LPS while otherwise remaining unchanged, which follows conflicting previous studies demonstrating that proinflammatory stimuli increased or had no impact on IDO2 mRNA [60, 61]. Overall, TDO expression did not increase in any brain region at either time point in response to LPS treatment, confirming previous reports demonstrating that TDO expression is up-regulated by glucocorticoids [62]. Together, these data confirm that IDO is the only rate-limiting kynurenine pathway enzyme up-regulated in response to peripheral LPS, demonstrating increased capacity for de novo kynurenine production during neuroinflammatory conditions.

The metabolism of kynurenine is physically compartmentalized within the brain $[24,53]$. Astrocytes express mainly KATs (not KMO) for conversion of kynurenine to KA $[63,64]$. Microglia express KMO (not KATs) that metabolizes kynurenine along the neurotoxic branch, leading to the potential formation of several neurotoxic metabolites $[65,66]$. Contemporary research suggests that the relative balance of these opposing metabolic branches, rather than simply changes in the levels of individual metabolites, constitutes the pathogenic potential of kynurenine metabolism [53]. Though LPS treatment increased GFAP expression and presumably astrocytic activation, there was only a significant increase in KATI expression in the hippocampus at $6 \mathrm{~h}$ post injections. Further, at $24 \mathrm{~h}$ post-LPS, KATI and KATII expression was reduced in the hippocampus. A previous expression study reported that LPS treatment did not affect KATII expression in the hippocampus and cortex [44], in contrast with our results in the hippocampus. The data reported here, suggesting that KAT expression (I and II) is generally unchanged by LPS treatment, are consistent with previous findings that demonstrate that whole brain KA concentration levels do not increase at $24 \mathrm{~h}$ postLPS treatment [23]. Instead, that same study demonstrated that peripheral LPS-induced elevations in central kynurenine result in increases in 3-HK, 3-HAA, and QA on the neurotoxic branch, mediated through $\mathrm{KMO}$ in 
microglia [23]. Interestingly, $\mathrm{KMO}$ was the only neurotoxic enzyme up-regulated following LPS treatment, while KYNU expression did not change and HAAO decreased expression following LPS treatment. In a previous study, at $4 \mathrm{~h}$ post-LPS, KMO expression decreased in the cortex, and at $24 \mathrm{~h}$ post-LPS, expression increased in both the cortex and hippocampus [44]. The experimental conditions, such as species (rats) and LPS dose $(0.25 \mathrm{mg} / \mathrm{kg})$, are likely contributors to the differences in expression kinetics between the two studies. In our study, it is noteworthy to consider the possibility that HAAO expression is down-regulated as a compensatory response to the increased expression of KMO and the potential increase in flux of metabolites through the neurotoxic branch. Interestingly, the disconnect between expression of microglia cell markers (Iba1, CD11b) and $\mathrm{KMO}$ provides further evidence that the functional cellular response is not necessarily accurately reflected by measuring standard microglial markers.

To further explore the effects of LPS on kynurenine metabolic balance within behaviorally relevant brain regions, we calculated ratios that (1) reflected IDO activity and up-regulation of overall kynurenine metabolism (kynurenine/tryptophan), (2) indicated the relative balance of kynurenine metabolism toward the neurotoxic branch (3-HK/KA), and (3) assessed serotonin turnover (5-HIAA/5-HT). Twenty-four hours post LPS was chosen as the sampling time point based on established behavioral kinetics [47]. Additionally, mRNA increases measured at $6 \mathrm{~h}$ post-LPS require time in order for protein translation to begin impacting metabolism relevant to behavior changes. In all of the regions assessed, the kynurenine/ tryptophan ratio was elevated as predicted based on IDO expression data and previously published data $[18,19,23$, 67]. However, assessment of the 3-HK/KA ratio, indicative of metabolism down the neurotoxic kynurenine branch, revealed that peripheral LPS treatment only increased this neurotoxic ratio in the dorsal and ventral hippocampus. Relevant to this, a recent study suggested that microglia in separate regions of the brain have functionally distinct characteristics, specifically that those in the hippocampus are more pro-inflammatory in nature [68]. As the main producers of neurotoxic kynurenine metabolites, these more immunovigilant hippocampal microglia might drive the region-specific shift toward neurotoxic metabolism observed here. Alternatively, it is possible that astrocytic production of KA may follow a region-dependent pattern, although this has yet to be directly investigated. Further, while not significant, increasing statistical power may actually reveal that the 3-HK/KA ratio decreases in the nucleus accumbens in response to peripheral LPS treatment. This will require additional future experiments. Interestingly, recent data collected from a chronic social defeat model, also associated with a peripheral immune response, demonstrated regional variation in the upregulation of central kynurenine and 3-HK production [69]. However, when brain region kynurenine metabolites were assessed in response to peripheral CD40 antibody treatment, which induces inflammation, both kynurenine and 3-HK were elevated in all of the regions assessed [70]. Previous studies have demonstrated that peripheral LPS treatment increases serotonin turnover in the whole brain [19]. In line with those data, the 5-HIAA/5-HT ratio was increased or trended to increase in all the regions assessed in this study, except the nucleus accumbens. Together, these data illustrate, for the first time, that brain kynurenine metabolism is regulated in a regionally distinct manner following peripheral immune challenge with LPS. Our recently published data support the notion that this neurotoxic shift is functionally relevant, as targeted deletion of the KMO gene protects mice from LPS-induced disruption in a hippocampal-dependent behavioral task [26].

As a control measure, tryptophan and kynurenine metabolites were assessed in the plasma $24 \mathrm{~h}$ after LPS treatment (Table 1). As predicted, LPS increased kynurenine, 3-HK, the kynurenine/tryptophan ratio, and the 3HK/KA ratio and did not change KA. Curiously, the tryptophan concentration increased as well, contrary to previous clinical and preclinical studies demonstrating that inflammation is associated with a decrease in tryptophan or 'tryptophan depletion' [16, 19, 71]. Further, there were no changes in 3-HAA or QA after LPS treatment, again differing from previous studies showing LPS elevates peripheral and central QA as well as central 3HAA [23, 71]. The current study is the first to explore kynurenine metabolic balance downstream of IDO in C57BL/6J inbred mice while previous studies were carried out in CD-1 outbred mice [23]. The dissociation between central and peripheral downstream kynurenine metabolites has also been observed in the clinic, when cerebrospinal fluid QA was elevated without an increase in plasma QA in hepatitis $C$ patients receiving interferon- $\alpha$ immunotherapy [72]. Importantly, our data revealed that even though kynurenine/tryptophan doubles in both the peripheral circulation and in each of the brain regions measured (supporting the notion of the periphery as a primary source of these amino acids), the manner in which kynurenine metabolism proceeds is not simply a function of substrate levels. The distinct 3-HK/KA ratios, in the face of uniform increases in kynurenine/tryptophan ratios, further underscore that downstream brain region metabolism is regionally discrete and locally regulated. Understanding how local metabolic changes impact the behaviors mediated by distinct brain regions remains an important area for future study. Together, these data demonstrate that peripheral LPS up-regulates the metabolism of tryptophan and kynurenine to $3-\mathrm{HK}$, in both the periphery and the brain. However, region-dependent regulation of kynurenine 
metabolic balance within the brain is potentially pertinent to the pathogenesis of depression and is a therapeutically relevant finding.

\section{Conclusions}

The main goal of this study was to provide a better understanding of the regional neuroinflammatory response following peripheral treatment with LPS. This response involved mRNA expression (Fig. 1) of proinflammatory cytokines (IL-1 $\beta, T N F \alpha$, IL-6), glia cell markers (CD11b, Iba1, GFAP), and kynurenine pathway enzymes (IDO, IDO2, TDO, KATI, KATII, KMO, KYNU, HAAO) at 6 and $24 \mathrm{~h}$ post LPS in relevant brain regions (hippocampus, amygdala, striatum). Additionally, kynurenine metabolite ratios (kynurenine/tryptophan, 3-HK/KA, 5-HIAA/5-HT) were calculated from previously collected data at $24 \mathrm{~h}$ post-LPS in relevant brain regions (dorsal and ventral hippocampus, central amygdala, nucleus accumbens). In general, these data demonstrated that in response to peripheral LPS treatment, pro-inflammatory cytokines and glia cell makers increase similarly throughout the brain. Kynurenine pathway enzymes have distinct temporal expression changes following LPS, which are region-independent; however, the metabolic balance is regulated in a regiondependent manner. Further analysis of the individual metabolite concentrations and additional brain regions will advance the understanding and potential implications of brain region-specific kynurenine metabolism. The possibility that individual depression-related behaviors are driven by regionally defined disruptions in kynurenine metabolism provides a specific target for future pharmacotherapy developments.

\begin{abstract}
Abbreviations
3-HAA, 3-hyroxyanthranilic acid; 3-HK, 3-hydroxykynurenine; 5-HIAA, 5hyrdoxyindoleacetic acid; $5-\mathrm{HT}$, serotonin; CD11b, cluster of differentiation molecule 11b; CSF, cerebrospinal fluid; fMRI, functional magnetic resonance imaging; GFAP, glial fibrillary acidic protein; HAAO, 3-hyroxyanthranilic acid dioxygenase; HPA, hypothalamic-pituitary-adrenal; IDO, indoleamine 2,3dioxygenase; i.p., intraperitoneal; Iba1, ionized calcium-binding adapter molecule 1; IFN- $a$, interferon- $\alpha$; IL-1 $\beta$, interleukin-1 $\beta$; IL-6, interleukin-6; KA, kynurenic acid; KAT, kynurenine aminotransferase; KMO, kynurenine 3monooxygenase; KYNU, kynureninase; LC/MS, liquid chromatography/mass spectrometry; LPS, lipopolysaccharide; MADRS, Montgomery-Asberg Depression Rating Scale; NMDAR, N-methyl-D-aspartate receptor; NO, nitric oxide; QA, quinolinic acid; RDoC, Research Domain Criteria; SEM, standard error of the mean; TDO, tryptophan 2,3-dioxygenase; TNFa, tumor necrosis factor $a$; UTHSCSA, University of Texas Health Science Center at San Antonio; $a_{7} n A C h R$, $a_{7}$ nicotinic acetylcholine receptor
\end{abstract}

\section{Acknowledgements}

A special thanks to Susan T. Weintraub, PhD and Xiaoli Gao, PhD at the University of Texas Health Science Center at San Antonio Mass Spectrometry Core Facilities for their assistance with the LC/MS method design, sample preparation, sample analysis, and data collection. Mass spectrometry analyses were conducted on instrumentation obtained with funding from the National Institutes of Health (1S100D016417-01 to STW).

\section{Funding}

This research was supported by funding from the National Institute of Mental Health (R01MH090127 and P30MH089868 to JOC; 1F31MH102070-01A1 to JP) the National Center for Advancing Translational Studies (UL1TR001120 to JOC), and the Norman Hackerman Advanced Research Program (003659-0010-2012 to JOC). The content is the sole the responsibility of the authors and does not necessarily represent the views of the National Institute of Mental Health, the National Center for Advancing Translational Studies or the National Institutes of Health. R01MH090127 funded data collection, data analysis, data interpretation, and writing of the manuscript. P30MH089868, UL1TR001120, and 003659-00102012 supported the design of the study, data analysis, and data interpretation. 1F31MH102070-01A1 funded data analysis, data interpretation, and writing of the manuscript.

\section{Availability of data and materials}

All raw data are clearly summarized in the body or supplemental figures of the manuscript. Even though the raw data used in the analyses presented here are not traditionally databased informatics, genomics, engineering dataset, they are freely available for review upon request from the corresponding author

\section{Authors' contributions}

JP carried out the tissue collection, organized and analyzed the data, and drafted the manuscript. LR helped to collect the samples and performed the LC/MS analysis. JOC conceived of the study, led the design and coordination of the study, and prepared the final manuscript. All authors read and approved the final manuscript.

\section{Competing interests}

Although unrelated to the material presented or discussed in this manuscript, Jason C. O'Connor, PhD has consulted for Lundbeck Research, USA, and Janssen Research and Development, LLC. Jennifer M. Parrott and Laney Redus declare that they have no competing interests.

\section{Consent for publication}

Not applicable.

\section{Ethics approval and consent to participate} Not applicable.

\section{Author details}

${ }^{1}$ Department of Pharmacology, School of Medicine, Center for Biomedical Neuroscience, University of Texas Health Science Center at San Antonio, 422D Medical Building MC-7764, 7703 Floyd Curl Drive, San Antonio, TX 78229-3900, USA. ²Department of Pharmacology, School of Medicine, University of Texas Health Science Center at San Antonio, 418D Medical Building MC-7764, 7703 Floyd Curl Drive, San Antonio, TX 78229-3900, USA ${ }^{3}$ Department of Pharmacology, School of Medicine, Center for Biomedical Neuroscience and Mood Disorders Translational Research Core, University of Texas Health Science Center at San Antonio, 216B Medical Building MC-7764, 7703 Floyd Curl Drive, San Antonio, TX 78229-3900, USA. ${ }^{4}$ Audie L. Murphy Memorial VA Hospital, South Texas Veterans Health System, 7400 Merton Minter, San Antonio, Texas 78229-4404, USA.

Received: 4 March 2016 Accepted: 20 May 2016

Published online: 27 May 2016

References

1. Moussavi S, Chatterji S, Verdes E, Tandon A, Patel V, Ustun B. Depression, chronic diseases, and decrements in health: results from the World Health Surveys. Lancet. 2007;370:851-8.

2. Capuron L, Miller AH. Cytokines and psychopathology: lessons from interferon-alpha. Biol Psychiatry. 2004;56:819-24.

3. Brydon L, Harrison NA, Walker C, Steptoe A, Critchley HD. Peripheral inflammation is associated with altered substantia nigra activity and psychomotor slowing in humans. Biol Psychiatry. 2008;63:1022-9.

4. Harrison NA, Brydon L, Walker C, Gray MA, Steptoe A, Critchley HD. Inflammation causes mood changes through alterations in subgenual cingulate activity and mesolimbic connectivity. Biol Psychiatry. 2009;66: 407-14. 
5. Eisenberger NI, Berkman ET, Inagaki TK, Rameson LT, Mashal NM, Irwin MR. Inflammation-induced anhedonia: endotoxin reduces ventral striatum responses to reward. Biol Psychiatry. 2010;68:748-54.

6. Grigoleit JS, Kullmann JS, Wolf OT, Hammes F, Wegner A, Jablonowski S, Engler H, Gizewski E, Oberbeck R, Schedlowski M. Dose-dependent effects of endotoxin on neurobehavioral functions in humans. PLoS One. 2011;6:e28330.

7. Felger JC, Alagbe O, Hu F, Mook D, Freeman AA, Sanchez MM, Kalin NH, Ratti E, Nemeroff CB, Miller AH. Effects of interferon-alpha on rhesus monkeys: a nonhuman primate model of cytokine-induced depression. Biol Psychiatry. 2007;62:1324-33.

8. Felger JC, Mun J, Kimmel HL, Nye JA, Drake DF, Hernandez CR, Freeman AA, Rye DB, Goodman MM, Howell LL, Miller AH. Chronic interferon-alpha decreases dopamine 2 receptor binding and striatal dopamine release in association with anhedonia-like behavior in nonhuman primates. Neuropsychopharmacology. 2013;38:2179-87.

9. Orsal AS, Blois SM, Bermpohl D, Schaefer M, Coquery N. Administration of interferon-alpha in mice provokes peripheral and central modulation of immune cells, accompanied by behavioral effects. Neuropsychobiology. 2008;58:211-22.

10. Hayley S, Scharf J, Anisman H. Central administration of murine interferonalpha induces depressive-like behavioral, brain cytokine and neurochemical alterations in mice: a mini-review and original experiments. Brain Behav Immun. 2013:31:115-27.

11. Konsman JP, Veeneman J, Combe C, Poole S, Luheshi GN, Dantzer R. Central nervous action of interleukin-1 mediates activation of limbic structures and behavioural depression in response to peripheral administration of bacterial lipopolysaccharide. Eur J Neurosci. 2008;28:2499-510.

12. O'Connor JC, Andre C, Wang Y, Lawson MA, Szegedi SS, Lestage J, Castanon N, Kelley KW, Dantzer R. Interferon-gamma and tumor necrosis factor-alpha mediate the upregulation of indoleamine 2,3-dioxygenase and the induction of depressive-like behavior in mice in response to bacillus Calmette-Guerin. J Neurosci. 2009;29:4200-9.

13. Raison CL, Capuron L, Miller AH. Cytokines sing the blues: inflammation and the pathogenesis of depression. Trends Immunol. 2006;27:24-31.

14. Polazzi E, Contestabile A. Reciprocal interactions between microglia and neurons: from survival to neuropathology. Rev Neurosci. 2002;13:221-42.

15. Block ML, Zecca L, Hong JS. Microglia-mediated neurotoxicity: uncovering the molecular mechanisms. Nat Rev Neurosci. 2007;8:57-69.

16. Dantzer R, O'Connor JC, Lawson MA, Kelley KW. Inflammation-associated depression: from serotonin to kynurenine. Psychoneuroendocrinology. 2011; 36:426-36.

17. Andre C, O'Connor JC, Kelley KW, Lestage J, Dantzer R, Castanon N. Spatio-temporal differences in the profile of murine brain expression of proinflammatory cytokines and indoleamine 2,3-dioxygenase in response to peripheral lipopolysaccharide administration. J Neuroimmunol. 2008;200:90-9.

18. O'Connor JC, Lawson MA, Andre C, Briley EM, Szegedi SS, Lestage J, Castanon N, Herkenham M, Dantzer R, Kelley KW. Induction of IDO by bacille Calmette-Guerin is responsible for development of murine depressive-like behavior. J Immunol. 2009;182:3202-12.

19. O'Connor JC, Lawson MA, Andre C, Moreau M, Lestage J, Castanon N, Kelley KW, Dantzer R. Lipopolysaccharide-induced depressive-like behavior is mediated by indoleamine 2,3-dioxygenase activation in mice. Mol Psychiatry. 2009;14:511-22.

20. Ganong AH, Cotman CW. Kynurenic acid and quinolinic acid act at Nmethyl-D-aspartate receptors in the rat hippocampus. J Pharmacol Exp Ther. 1986;236:293-9.

21. Hilmas C, Pereira EF, Alkondon M, Rassoulpour A, Schwarcz R, Albuquerque EX. The brain metabolite kynurenic acid inhibits alpha7 nicotinic receptor activity and increases non-alpha7 nicotinic receptor expression: physiopathological implications. J Neurosci. 2001;21:7463-73.

22. Stone TW, Perkins MN. Quinolinic acid: a potent endogenous excitant at amino acid receptors in CNS. Eur J Pharmacol. 1981;72:411-2.

23. Walker AK, Budac DP, Bisulco S, Lee AW, Smith RA, Beenders B, Kelley KW, Dantzer R. NMDA receptor blockade by ketamine abrogates lipopolysaccharide-induced depressive-like behavior in C57BL/6 J mice. Neuropsychopharmacology. 2013;38:1609-16.

24. Parrott JM, O'Connor JC. Kynurenine 3-monooxygenase: an influentia mediator of neuropathology. Front Psychiatry. 2015;6:116.

25. Salazar A, Gonzalez-Rivera BL, Redus L, Parrott JM, O'Connor JC. Indoleamine 2,3-dioxygenase mediates anhedonia and anxiety-like behaviors caused by peripheral lipopolysaccharide immune challenge. Horm Behav. 2012;62:202-9.

26. Heisler JM, O'Connor JC. Indoleamine 2,3-dioxygenase-dependent neurotoxic kynurenine metabolism mediates inflammation-induced deficit in recognition memory. Brain Behav Immun. 2015;50:115-24.

27. Wichers MC, Koek GH, Robaeys G, Verkerk R, Scharpe S, Maes M. IDO and interferon-alpha-induced depressive symptoms: a shift in hypothesis from tryptophan depletion to neurotoxicity. Mol Psychiatry. 2005;10:538-44.

28. Raison CL, Dantzer R, Kelley KW, Lawson MA, Woolwine BJ, Vogt G, Spivey JR, Saito K, Miller AH. CSF concentrations of brain tryptophan and kynurenines during immune stimulation with IFN-alpha: relationship to CNS immune responses and depression. Mol Psychiatry 2010;15:393-403.

29. Insel T, Cuthbert B, Garvey M, Heinssen R, Pine DS, Quinn K, Sanislow C, Wang P. Research domain criteria (RDoC): toward a new classification framework for research on mental disorders. Am J Psychiatry. 2010;167:748-51.

30. Insel TR. The NIMH Research Domain Criteria (RDoC) Project: precision medicine for psychiatry. Am J Psychiatry. 2014;171:395-7.

31. Reichenberg A, Yirmiya R, Schuld A, Kraus T, Haack M, Morag A, Pollmacher T. Cytokine-associated emotional and cognitive disturbances in humans. Arch Gen Psychiatry. 2001;58:445-52.

32. Krabbe KS, Reichenberg A, Yirmiya R, Smed A, Pedersen BK, Bruunsgaard H. Low-dose endotoxemia and human neuropsychological functions. Brain Behav Immun. 2005;19:453-60.

33. Inagaki TK, Muscatell KA, Irwin MR, Cole SW, Eisenberger NI. Inflammation selectively enhances amygdala activity to socially threatening images. Neuroimage. 2012;59:3222-6.

34. Muscatell KA, Dedovic K, Slavich GM, Jarcho MR, Breen EC, Bower JE, Irwin MR, Eisenberger NI. Greater amygdala activity and dorsomedial prefrontalamygdala coupling are associated with enhanced inflammatory responses to stress. Brain Behav Immun. 2015;43:46-53.

35. Konsman JP, Kelley K, Dantzer R. Temporal and spatial relationships between lipopolysaccharide-induced expression of Fos, interleukin-1 beta and inducible nitric oxide synthase in rat brain. Neuroscience. 1999;89:535-48.

36. Drevets WC, Price JL, Furey ML. Brain structural and functional abnormalities in mood disorders: implications for neurocircuitry models of depression. Brain Struct Funct. 2008;213:93-118.

37. Gal EM, Sherman AD. L-kynurenine: its synthesis and possible regulatory function in brain. Neurochem Res. 1980;5:223-39.

38. Franklin KBJ, Paxinos G. The mouse brain in sterotaxic coordinates. 3rd ed. New York, NY: Academic Press; 2007.

39. Lawson LJ, Perry VH, Dri P, Gordon S. Heterogeneity in the distribution and morphology of microglia in the normal adult mouse brain. Neuroscience. 1990;39:151-70.

40. Buttini M, Limonta S, Boddeke HW. Peripheral administration of lipopolysaccharide induces activation of microglial cells in rat brain. Neurochem Int. 1996:29:25-35.

41. Okuno E, Nakamura M, Schwarcz R. Two kynurenine aminotransferases in human brain. Brain Res. 1991;542:307-12.

42. Corona AW, Norden DM, Skendelas JP, Huang Y, O'Connor JC, Lawson M, Dantzer R, Kelley KW, Godbout JP. Indoleamine 2,3-dioxygenase inhibition attenuates lipopolysaccharide induced persistent microglial activation and depressive-like complications in fractalkine receptor (CX(3)CR1)-deficient mice. Brain Behav Immun. 2013;31:134-42.

43. Dinel AL, Andre C, Aubert A, Ferreira G, Laye S, Castanon N. Lipopolysaccharide-induced brain activation of the indoleamine 2,3dioxygenase and depressive-like behavior are impaired in a mouse model of metabolic syndrome. Psychoneuroendocrinology. 2014;40:48-59.

44. Connor TJ, Starr N, O'Sullivan JB, Harkin A. Induction of indolamine 2,3-dioxygenase and kynurenine 3-monooxygenase in rat brain following a systemic inflammatory challenge: a role for IFN-gamma? Neurosci Lett. 2008;441:29-34.

45. Bluthe RM, Pawlowski M, Suarez S, Parnet P, Pittman Q, Kelley KW, Dantzer R. Synergy between tumor necrosis factor alpha and interleukin-1 in the induction of sickness behavior in mice. Psychoneuroendocrinology. 1994;19:197-207.

46. Kozak W, Conn CA, Kluger MJ. Lipopolysaccharide induces fever and depresses locomotor activity in unrestrained mice. Am J Physiol. 1994; 266:R125-35. 
47. Dantzer R, O'Connor JC, Freund GG, Johnson RW, Kelley KW. From inflammation to sickness and depression: when the immune system subjugates the brain. Nat Rev Neurosci. 2008;9:46-56.

48. Lenczowski MJ, Bluthe RM, Roth J, Rees GS, Rushforth DA, van Dam AM, Tilders FJ, Dantzer R, Rothwell NJ, Luheshi GN. Central administration of rat IL-6 induces HPA activation and fever but not sickness behavior in rats. Am J Physiol. 1999;276:R652-8.

49. Quan N, Banks WA. Brain-immune communication pathways. Brain Behav Immun. 2007;21:727-35.

50. Banks WA, Kastin AJ, Broadwell RD. Passage of cytokines across the bloodbrain barrier. Neuroimmunomodulation. 1995;2:241-8.

51. Sugaya K, Chou S, Xu SJ, McKinney M. Indicators of glial activation and brain oxidative stress after intraventricular infusion of endotoxin. Brain Res Mol Brain Res. 1998;58:1-9.

52. Ito D, Imai Y, Ohsawa K, Nakajima K, Fukuuchi Y, Kohsaka S. Microgliaspecific localisation of a novel calcium binding protein, Iba1. Brain Res Mol Brain Res. 1998:57:1-9.

53. Schwarcz R, Bruno JP, Muchowski PJ, Wu HQ. Kynurenines in the mammalian brain: when physiology meets pathology. Nat Rev Neurosci. 2012;13:465-77.

54. Brahmachari S, Fung YK, Pahan K. Induction of glial fibrillary acidic protein expression in astrocytes by nitric oxide. J Neurosci. 2006;26:4930-9.

55. Roy A, Fung YK, Liu X, Pahan K. Up-regulation of microglial CD11b expression by nitric oxide. J Biol Chem. 2006;281:14971-80.

56. Rios C, Santamaria A. Quinolinic acid is a potent lipid peroxidant in rat brain homogenates. Neurochem Res. 1991;16:1139-43.

57. Santamaria A, Santamaria D, Diaz-Munoz M, Espinoza-Gonzalez V, Rios C Effects of $\mathrm{N}$ omega-nitro-L-arginine and L-arginine on quinolinic acidinduced lipid peroxidation. Toxicol Lett. 1997;93:117-24.

58. Perez-Severiano F, Escalante B, Rios C. Nitric oxide synthase inhibition prevents acute quinolinate-induced striatal neurotoxicity. Neurochem Res. 1998:23:1297-302.

59. Fatokun AA, Hunt NH, Ball HJ. Indoleamine 2,3-dioxygenase 2 (IDO2) and the kynurenine pathway: characteristics and potential roles in health and disease. Amino Acids. 2013;45:1319-29.

60. Ball HJ, Sanchez-Perez A, Weiser S, Austin CJ, Astelbauer F, Miu J, McQuillan JA, Stocker R, Jermiin LS, Hunt NH. Characterization of an indoleamine 2,3-dioxygenase-like protein found in humans and mice. Gene. 2007:396:203-13.

61. Divanovic S, Sawtell NM, Trompette A, Warning Jl, Dias A, Cooper AM, Yap GS, Arditi M, Shimada K, Duhadaway JB, et al. Opposing biological functions of tryptophan catabolizing enzymes during intracellular infection. J Infect Dis. 2012;205:152-61.

62. Danesch U, Hashimoto S, Renkawitz R, Schutz G. Transcriptional regulation of the tryptophan oxygenase gene in rat liver by glucocorticoids. J Biol Chem. 1983;258:4750-3.

63. Kiss C, Ceresoli-Borroni G, Guidetti P, Zielke CL, Zielke HR, Schwarcz R. Kynurenate production by cultured human astrocytes. J Neural Transm. 2003;110:1-14

64. Guidetti P, Hoffman GE, Melendez-Ferro M, Albuquerque EX, Schwarcz R. Astrocytic localization of kynurenine aminotransferase II in the rat brain visualized by immunocytochemistry. Glia. 2007;55:78-92.

65. Heyes MP, Achim CL, Wiley CA, Major EO, Saito K, Markey SP. Human microglia convert I-tryptophan into the neurotoxin quinolinic acid. Biochem J. 1996:320(Pt 2):595-7.

66. Guillemin GJ, Smythe G, Takikawa O, Brew BJ. Expression of indoleamine 2,3-dioxygenase and production of quinolinic acid by human microglia, astrocytes, and neurons. Glia. 2005;49:15-23.

67. Lawson MA, Parrott JM, McCusker RH, Dantzer R, Kelley KW, O'Connor JC Intracerebroventricular administration of lipopolysaccharide induces indoleamine2,3-dioxygenase-dependent depression-like behaviors. J Neuroinflammation. 2013;10:87.

68. Zeisel A, Munoz-Manchado AB, Codeluppi S, Lonnerberg P, La Manno G, Jureus A, Marques S, Munguba H, He L, Betsholtz C, et al. Brain structure. Cell types in the mouse cortex and hippocampus revealed by single-cell RNA-seq. Science. 2015;347:1138-42.

69. Fuertig $R$, Azzinnari $D$, Bergamini $G$, Cathomas F, Sigrist $H$, Seifritz $E_{\text {, }}$ Vavassori S, Luippold A, Hengerer B, Ceci A, Pryce CR. Mouse chronic social stress increases blood and brain kynurenine pathway activity and fear behaviour: both effects are reversed by inhibition of indoleamine 2,3-dioxygenase. Brain Behav Immun. 2016;54:59-72.
70. Cathomas F, Fuertig R, Sigrist H, Newman GN, Hoop V, Bizzozzero M, Mueller A, Luippold A, Ceci A, Hengerer B, et al. CD40-TNF activation in mice induces extended sickness behavior syndrome co-incident with but not dependent on activation of the kynurenine pathway. Brain Behav Immun. 2015;50:125-40.

71. Saito K, Markey SP, Heyes MP. Effects of immune activation on quinolinic acid and neuroactive kynurenines in the mouse. Neuroscience. 1992;51:25-39.

72. Pettitt SJ, Liang Q, Rairdan XY, Moran JL, Prosser HM, Beier DR, Lloyd KC, Bradley A, Skarnes WC. Agouti C57BL/6N embryonic stem cells for mouse genetic resources. Nat Methods. 2009;6:493-5.

\section{Submit your next manuscript to BioMed Central and we will help you at every step:}

- We accept pre-submission inquiries

- Our selector tool helps you to find the most relevant journal

- We provide round the clock customer support

- Convenient online submission

- Thorough peer review

- Inclusion in PubMed and all major indexing services

- Maximum visibility for your research

Submit your manuscript at www.biomedcentral.com/submit
Biomed Central 